\title{
Cancelling thermal cross-talk effects in photonic integrated circuits
}

\author{
Maziyar Milanizadeh, Douglas Aguiar, Andrea Melloni and Francesco Morichetti
}

\begin{abstract}
Thermal actuators are among the most consolidated and widespread devices for the active control of photonic integrated circuits (PICs). As a main drawback, mutual thermal crosstalk among actuated devices integrated onto the same photonic chip can affect the working point of the PIC and can reduce the efficiency of automated tuning and calibration procedures. In this work, a strategy to cancel out the effects of the phase coupling induced by thermal crosstalk is presented. In our technique, that we named Thermal Eigenmode Decomposition (TED), all the actuators of the PIC are controlled simultaneously according to the eigensolution of the thermally coupled system. The effectiveness of the TED method is validated by numerical simulations and experiments carried out on coupled microring resonator (MRR) and switch fabrics of Mach-Zehnder interferometers (MZIs). With respect to individual control of phase actuators, where thermal crosstalk can hinder the convergence of automated tuning algorithms, with the TED technique convergence is always reached, requires a lower number of iterations, and is less sensitive to the initial state of the PIC. The proposed TED method can be applied to generic tuning and locking algorithm, can be employed in arbitrary PIC architectures and its validity can be extended to systems where phase coupling is induced by other physical effects, such as mutual mechanical stress and electromagnetic coupling among RF lines.
\end{abstract}

Index Term - Control algorithms, integrated photonics, thermal cross talk, phase coupling, tuning and locking

\section{INTRODUCTION}

$\mathrm{P}_{\mathrm{c} i n}^{\mathrm{h}}$ hotonic integrated circuits (PICs) are evolving towards onchip reconfigurable architectures and general purpose programmable photonic processors, enabling the implementation of many different functionalities on-demand $[1,2,3,4]$. These schemes rely on the use of a large number of optical interferometers, such as Mach-Zehnder interferometers (MZI) and microring resonators (MRRs), whose individual working point is inherently related to the phase delay between the interfering optical beams. Therefore, any kind of phase perturbation may substantially affect the overall behavior of the PIC.

This work was partially supported by the European Commission through the H2020 project ICT-STREAMS (Contract No. 688172) and by Fondazione Cariplo (grant n. 2016-0881, ACTIO).

The authors are with the Department of Electronics, Information and Bioengineering, University Politecnico di Milano, Piazza Leonardo da vinci, 32, 20133, Milano, Italy. (email: maziyar.milanizadeh@polimi.it)
Phase perturbations are naturally originated by tolerances of the fabrication process, owing to the sensitivity of the effective index of the optical waveguides to nanometer-scale dimensional variations [5, 6]. Moreover, time-varying phase drifts may also be caused [6] by temperature changes induced by environmental thermal fluctuations or by heat sources integrated onto the same photonic chip [7, 8, 9].

To compensate against phase errors as well as to reconfigure and stabilize the working point of a PIC, actuators capable of controlling actively the phase in optical waveguides are required $[10,11,12,13]$. Thermal actuators are a wellestablished approach [14, 15] however, thermal actuators can induce mutual thermal crosstalk between neighbor actuated waveguides and thus can impair the efficiency of control procedures employed for PIC tuning and stabilization.

Several solutions have been proposed to mitigate thermal cross-talk on a photonic chip. Thermal isolation trenches allow the localization of the heat around the actuated waveguide and can also improve the heater efficiency [2] [16]. However, this approach puts some constraints on the layout and footprint of the PIC and is not exploitable for closely spaced devices, as in the case of coupled MRRs. Athermal optical waveguides have been demonstrated by coating the waveguide core with a material with a negative thermooptic coefficient (TOC) $[17,18]$ yet, reducing the temperature sensitivity of the waveguide prevents from the possibility to perform active thermal tuning. Thermally self-compensating Mach-Zehnder interferometer (MZI) were fabricated by adopting different geometries for the two arms of the interferometer [19] or operating on orthogonal polarization states [20]. While being effective, this approach is circuit-specific, and hence hardly scalable to generic PIC architectures.

In this contribution we present a novel method, that we named Thermal Eigenmode Decomposition (TED), which is not a control algorithm but a technique that can be adopted in control algorithms to cancel out the effects of thermal crosstalk in arbitrary PICs where thermal crosstalk is indeed physically present. In other words, the TED concept is neither circuitspecific, nor algorithm specific. In Sec. II we introduce the main concepts and the theory of the TED method, together with an example of tuning algorithm where it can be used. To demonstrate the effectiveness of the TED method on generic PICs, numerical simulations are presented in Sec III on two well-known architectures, namely a coupled MRR filter and an MZI switch fabric. Experimental results on a coupled MRR filter are reported in Sec. IV, providing a clear evidence of the benefits of the TED method, with respect to conventional control strategies used for the tuning and wavelength locking of 

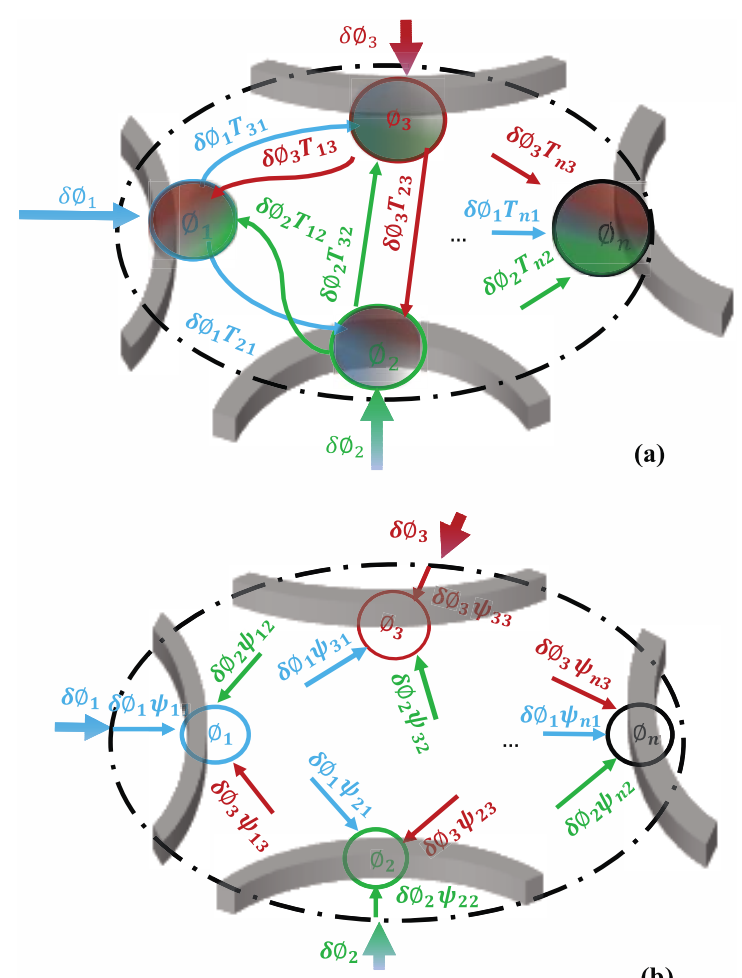

(b)

Fig. 1: (a) Schematic representation of a PIC integrating $N$ phase actuators in the presence of phase coupling induced by thermal cross-talk. (b) TED concept: the effects of thermal cross-talk are cancelled by simultaneously driving all the coupled actuators according to the eigenmodes of the thermally coupled system.

a PIC affected by thermal crosstalk. In Sec. IV we discuss some additional details on the use of the TED method in generic photonic architectures and a concluding section $\mathrm{V}$ summarizes the main achievements of this work.

\section{THERMAL EIGENMODE DECOMPOSITION}

To illustrate the concept of the TED method, let us consider the schematic of Fig. 1(a) showing an arbitrary PIC consisting of $N$ optical waveguides with a thermal actuator integrated in each of them. The status of the circuit is identified by the phase vector $\boldsymbol{\Phi}=\left[\begin{array}{lll}\Phi_{1} & \ldots & \Phi_{N}\end{array}\right]^{\mathrm{T}}$, where $\Phi_{n}$ is the current phase in the $n$-th waveguide. When an electrical power is applied to the $n$ th actuator, it is expected to introduce a desired phase change $\delta \Phi_{n}$ to the $n$-th waveguide where the actuator is integrated, with no effects on the surrounding waveguides. However, due to thermal cross-talk, some phase perturbations are also introduced in the other waveguides. Considering Fig. 1(a) the actual phase shifts $\delta \widetilde{\boldsymbol{\Phi}}=\left[\begin{array}{llll}\delta \widetilde{\Phi}_{1} & \ldots & \delta \widetilde{\Phi}_{N}\end{array}\right]^{\mathrm{T}}$ induced in each waveguide is given by

$$
\delta \widetilde{\boldsymbol{\Phi}}=\left(\begin{array}{ccccc}
T_{11} & T_{12} & T_{13} & \cdots & T_{1 n} \\
T_{21} & T_{22} & T_{23} & \cdots & T_{2 n} \\
T_{31} & T_{32} & T_{33} & \cdots & T_{3 n} \\
\vdots & \vdots & \vdots & \ddots & \vdots \\
T_{n 1} & T_{n 2} & T_{n 3} & \cdots & T_{n n}
\end{array}\right) \delta \boldsymbol{\Phi}=\mathbf{T} \delta \boldsymbol{\Phi}
$$

where $\delta \boldsymbol{\Phi}=\left[\begin{array}{llll}\delta \Phi_{1} & \ldots & \delta \Phi_{N}\end{array}\right]^{\mathrm{T}}$ is the desired phase shift and $\mathbf{T}$ is the phase coupling matrix taking into account all the self (diagonal) and cross (off-diagonal) phase shift contributions.
Without loss of generality, all the diagonal terms can be assumed unitary, that is $T_{n n}=1$. The phase coupling coefficient $T_{n m}$ between the $n$-th actuator and the $m$-th waveguide depends on the PIC topology, photonic platform and not on the status of the circuit.

When applying control algorithms for the automatic tuning of the PIC, mutual phase perturbation among thermal actuators must be compensated. However, individual corrections of each phase change $\delta \widetilde{\Phi}_{n}$ is not an effective method for controlling the system. First, this would require for each actuator the need for post-compensating the thermal crosstalk that has been introduced by other actuators at previous steps, thus leading to a substantial increase of the number of iterations required to steer the PIC to the desired working point. Second, as shown in the following of this paper, the convergence of the algorithm itself could be severely impaired.

To circumvent this issue, the proposed TED method provides a strategy to cancel out the unwanted effects of thermal crosstalk on the actual phase shift applied to the optical waveguides. Mathematically, the concept is extremely simple because it is essentially a coordinate transformation, mapping the phase variables $\boldsymbol{\Phi}$, which are (thermally) coupled by the $\mathbf{T}$ matrix, into a suitable set of uncoupled phase variables $\boldsymbol{\Psi}=\left[\begin{array}{llll}\delta \Psi_{1} & \ldots & \delta \Psi_{N}\end{array}\right]^{\mathrm{T}}$, for which the phase coupled matrix $\mathbf{T}_{\mathrm{D}}$ becomes diagonal. Assuming $\mathbf{T}$ to be diagonalizable (see Sec. $\mathrm{V}$ for more details on the requirements of the $\mathbf{T}$ matrix), we can write

$$
\delta \widetilde{\boldsymbol{\Phi}}=\mathbf{P T}_{\mathrm{D}} \mathbf{P}^{-1} \delta \boldsymbol{\Phi}
$$

where $\mathbf{P}$ is a matrix whose columns are linearly independent eigen-vectors of $\mathbf{T}, \mathbf{T}_{\mathrm{D}}$ is the diagonal matrix containing the corresponding eigen-values, and $\mathbf{P}^{-1}$ is the inverse matrix of $\mathbf{P}$. Multiplying both sides by $\mathbf{P}^{-1}$ we obtain

$$
\delta \widetilde{\boldsymbol{\Psi}}=\mathbf{T}_{\mathrm{D}} \delta \boldsymbol{\Psi},
$$

where

$$
\delta \Psi=\mathbf{P}^{-1} \delta \Phi
$$

is the phase shift imparted to the transformed phase variables $\boldsymbol{\Psi}$. Since $\mathbf{T}_{\mathrm{D}}$ is diagonal, any change in each element of vector $\boldsymbol{\Psi}$ does not affect the other elements. In other words, the elements $\boldsymbol{\Psi}_{n}=\mathbf{P}_{n}^{-1} \boldsymbol{\Phi}$, where $\mathbf{P}_{n}^{-1}$ is the $n$-th row of the $\mathbf{P}^{-1}$ matrix, identify orthogonal directions in a transformed phase space, enabling to apply uncoupled, and hence well controllable, phase modifications to the system.

From a physical stand point, the TED method implies that all the actuators, that are thermally coupled, need to be simultaneously modified to apply the desired change. The weights are defined by the eigenmodes of the thermally coupled system, that is by the rows of the $\mathbf{P}$ matrix.

As expected and shown by simulations reported in this work, the best performance of the TED method is achieved when the phase coupling matrix $\mathbf{T}$ is precisely known. However, accurate information on thermal crosstalk is hardly available in practical cases. Noteworthy, our results show that the TED provides significant performance improvement with respect to individual tuning of thermal actuators, even when partial knowledge of the $\mathbf{T}$ matrix is available, for instance from empirical models, numerical thermal simulations, and experimental data. 
Once phase mapping through TED is performed, any tuning and locking algorithm can be implemented by using the transformed coordinates $\boldsymbol{\Psi}$ as phase state variables of the system. Including feedback iterative approaches (like [11] [12]), multi degree of freedom (DOF) stabilization methods [21], linear state-space control systems [22], dithering technique locking approaches [23] [24] .

These concepts have been employed in the numerical and experimental examples reported in the following sections.

\section{NUMERICAL RESUlTS}

To show the effectiveness of the TED method on generic photonic architectures, in this section we present the results of numerical simulations carried out on two different PICs, namely a coupled MRR filter and an MZI based switch fabric. Coupled MRR filters are widely used because of their highlyselective frequency response, which also makes their performance very sensitive to fabrication tolerances, temperature drifts and laser wavelength fluctuations. MZI fabrics find applications for the realization of compact, broadband, high-port-count optical switches. In both cases, thermal actuation is one of the mostly used approach for active tuning and configuration, and thermal crosstalk effects must be counteracted to guarantee reliable control and high performance.

Here we describe a simple closed-loop algorithm (which uses TED technique to modify the actuators) that can be applied to generic PIC architecture, as confirmed by numerical and experimental results reported in the following of this paper. Referring to the flow diagram of Fig. 2, phase modifications $\delta \Psi_{n}$ are applied to match a target goal function, which can be for instance the minimization (or maximization) of the output optical power at certain optical ports. At each iteration, a step along $\boldsymbol{\Psi}_{n}$ is followed by calculating the corresponding phase vector $\boldsymbol{\Phi}$, providing the phase shift to be simultaneously given by each of the actuators which is obtained as $\boldsymbol{\Phi}=\mathbf{P \Psi}$. After each step $\delta \Psi_{n}$ is performed, the error function is evaluated. At this point, if progress is along the desired path (minimizing or maximizing the reading point value in this example), following steps are repeated in the same direction, otherwise direction is reversed by inverting the sign of $\delta \Psi_{n}$. Once the PIC has reached the target configuration, the algorithm can be let to operate realizing a locking scheme, capable of maintaining the tuned condition in the event of unwanted perturbation, such as temperature fluctuations as well as drifts of the laser wavelength. Adaptive phase steps can also be used to find the best trade off between the converge speed of the tuning process and the residual oscillation introduced in system during the locking phase.

Optical power at a specific port is defined as the cost function for these analyses. This means algorithm is evaluated based on the convolution of modulated signal with transfer function of the filter. It allows the algorithm to push the filter matching the spectrum of the signal to obtain the best isolation (or negligible perturbation) for a specific channel. Other metrics like bit error rate (BER), eye diagram opening, amplitude of channels labels [25] or any other relevant parameter can be used as cost function depending on the application.

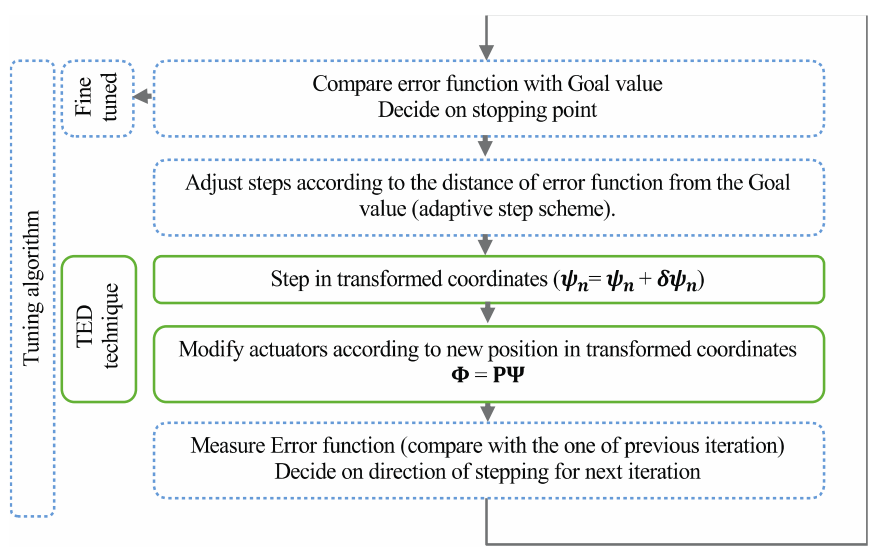

Fig. 2: Block diagram of an example tuning algorithm using TED technique to modify actuators. At each iteration a step taken in transformed coordinate $\Psi_{n}$ is translated to variation of actuators by $\mathbf{\Phi}=\mathbf{P} \Psi$. Error function is evaluated and compared with the one of previous iteration to track the progress. If progress is not along the desired path, direction of movement is reversed simply by inversing the sign of $\delta \Psi_{n}$. At the end of each cycle error function is compared with the goal to define the stopping point.

\section{A. Coupled MRR filter}

Figure 3(a) shows the schematic of a coupled MRR filter. A thermal actuator (in red) is integrated inside each resonator to modify the round-trip phase $\Phi_{i}$. Here we consider a $3^{\text {rd }}$ order MRR architecture, but the proposed approach can be generalized to structures made of an arbitrary number of resonators. Conventional tuning methods for this kind of filters (hereinafter referred to as "individual tuning") exploit sequential sweeping of the individual resonance of each MRR for aligning it to the desired wavelength [10] [12]. However, we show that in the presence of thermal crosstalk, not only these approaches typically require a higher number of iterations, but they can also suffer from instability issues and may not even converge. In contrast, our results show that in all the considered cases convergence is always obtained when TED is applied.

For simplicity, we make the assumption that thermal crosstalk induces equal phase coupling $\mu$ between neighbor MRRs, while there is no phase coupling between the first and the third MRR. In this case, the phase coupling matrix is

$$
\mathbf{T}=\left(\begin{array}{lll}
1 & \mu & 0 \\
\mu & 1 & \mu \\
0 & \mu & 1
\end{array}\right)
$$

whose eigenvectors

$$
\mathbf{P}_{1}=\left(\begin{array}{c}
-1 \\
0 \\
1
\end{array}\right), \quad \mathbf{P}_{2}=\left(\begin{array}{c}
1 \\
\sqrt{2} \\
1
\end{array}\right), \quad \mathbf{P}_{3}=\left(\begin{array}{c}
1 \\
-\sqrt{2} \\
1
\end{array}\right)
$$

are the columns of matrix $\mathbf{P}$. However, as discussed in Sec. V, this assumption does not limit the generality of the approach. By inverting the $\mathbf{P}$ matrix, the orthogonal phase coordinates are calculated as given in (4), that are 


$$
\begin{gathered}
\delta \Psi_{1}=\mathbf{P}_{1}^{-1} \delta \boldsymbol{\Phi}=\left(\begin{array}{ccc}
-\frac{1}{2} & 0 & \frac{1}{2}
\end{array}\right) \delta \boldsymbol{\Phi} \\
\delta \Psi_{2}=\mathbf{P}_{2}^{-1} \delta \boldsymbol{\Phi}=\left(\begin{array}{lll}
\frac{1}{4} & \frac{\sqrt{2}}{4} & \frac{1}{4}
\end{array}\right) \delta \boldsymbol{\Phi} \\
\delta \Psi_{3}=\mathbf{P}_{3}^{-1} \delta \boldsymbol{\Phi}=\left(\begin{array}{lll}
\frac{1}{4} & -\frac{\sqrt{2}}{4} & \frac{1}{4}
\end{array}\right) \delta \boldsymbol{\Phi} .
\end{gathered}
$$

At each iteration, a step is taken along these new coordinates and the related phase changes $\delta \boldsymbol{\Phi}$ of each MRR are calculated as $\delta \boldsymbol{\Phi}=\mathbf{P} \delta \boldsymbol{\Psi}$.

As a case study, we consider a $3^{\text {rd }}$ order MRR filter with a free spectral range (FSR) of $50 \mathrm{GHz}$ and bandwidth equal to 6.5 $\mathrm{GHz}$. Random phase errors as large as $\pm \pi / 4( \pm 6.25 \mathrm{GHz})$ are intentionally introduced among the resonances of the MRRs,

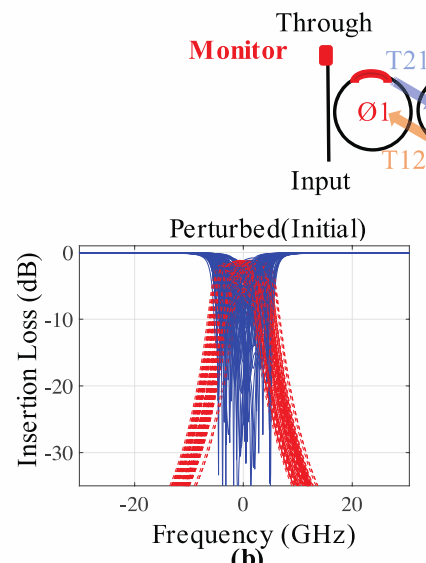

(b)

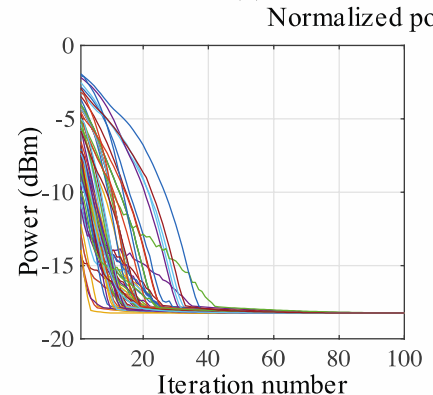

(d)

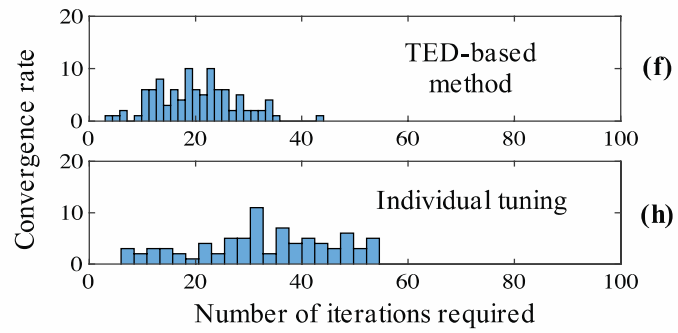

Fig. 3: Numerical simulations of the tuning of a $3^{\text {rd }}$ order coupled MRR filter in the presence of thermal crosstalk $(\mu=0.15)$. (a) Schematic of the filter presenting assumed phase coupling between resonators. (b) Spectral response in the initial state of the filter (100 configurations, up to $\pm \pi / 4$ random phase perturbations). (c) Through (blue-solid) and Drop (reddashed) port transmission after the convergence of the TED-based tuning algorithm. Convergence curves showing the normalized power at Through port of the filter during (d) the TED-based tuning and (e) individual tuning of each MRR. Histogram showing the required number of iterations to reach the goal point using (f) the TED-based tuning or (h) individual tuning of each MRR for converged cases.

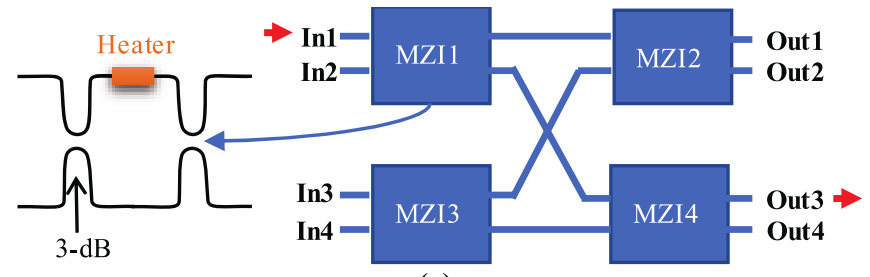

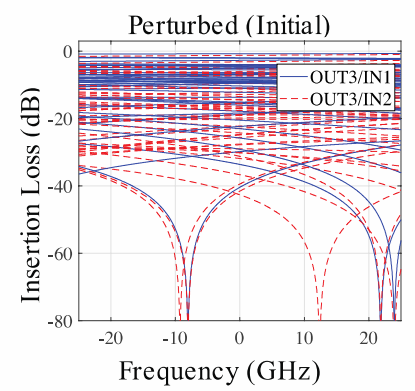

(b)

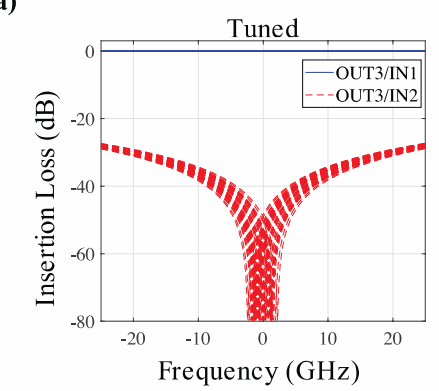

(c)

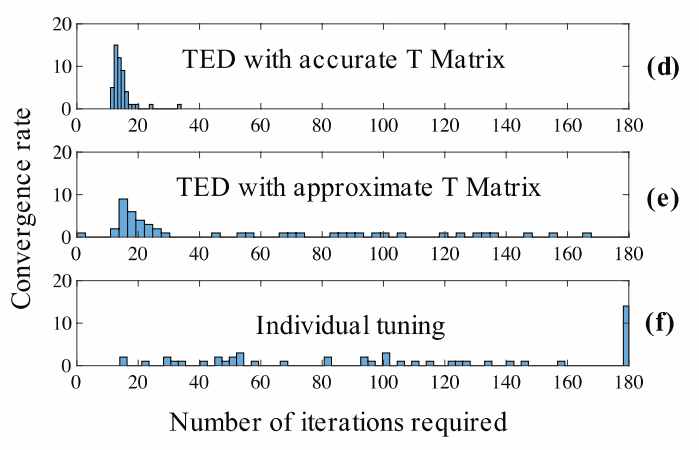

Fig. 4: Numerical simulation of the tuning of a $4 \times 4$ MZI switch fabric in the presence of thermal crosstalk $(\mu=0.18$ ). (a) Schematic of circuit. (b) Initial state of the switch (50 configurations, up to $\pm \pi / 4$ random phase perturbations); (c) Frequency domain transmission Out3/In1 (blue-solid) and Out3/In2 (red-dashed) after the convergence of the TED-based tuning algorithm. Histogram showing the required number of iterations to set the routed path of the switch fabric by using (d) the TED-based tuning using accurate phase coupling matrix (T), (e) TED-based tuning using approximate phase coupling matrix and (f) individual tuning of each MZI.

resulting in the 100 random initial configurations shown in Fig.3(b). At the Input port, an optical signal modulated according to a $5 \mathrm{Gbit} / \mathrm{s}$ on-off keying (OOK) intensity modulation is used. To model thermal crosstalk, a phase coupling coefficient $\mu=0.15$ is assumed between actuators.

To point out the benefit of our approach, the filter was tuned by using the algorithm of Fig. 2 to minimize the optical power at the Through port, using as phase variables the transformed coordinated $\boldsymbol{\Psi}$ by the TED method and the round-trip phases $\boldsymbol{\Phi}$ for the individual tuning method, respectively. In both cases, we assumed the same initial conditions of Fig. 3(b) for the perturbed filter and the same phase step-size of $0.015 \mathrm{rad}$.

Figure 3(c) shows the frequency domain response of the filter for all the considered cases after the implementation of TEDbased algorithm. Convergence to almost overlapping spectra at the Through (blue curves) and Drop (red curves) is achieved regardless of the initial perturbed state of the filter. The convergence curves in Fig. 3(d), providing the optical power at the Through port, show that less than 50 iterations are required to tune filter to the desired state, where the isolation at the Through port is about $18 \mathrm{~dB}$. In contrast, when individual tuning is used (see Fig. 3(e)), convergence is not guaranteed 
and, depending on the initial state of the filter, residual oscillations may appear that prevent the algorithm to bring the filter to the target state. In the considered example, $10 \%$ of trails did not converge to the maximum isolation. Restricting the analysis to the cases where convergence is achieved, the average number of required iterations in individual tuning is higher than the ones in the TED-based method. Histogram in Fig. 3(f) shows the required number of iterations to reach goal point using the TED-based tuning while Fig.3(h) presents results for individual tuning of each MRR. This increment in average and standard deviation indicates the dependence of convergence on the starting points because of thermal crosstalk.

\section{B. MZI switch fabric}

The second architecture that we considered is the $4 \times 4$ MZI arrangement depicted in Fig. 4(a). It consists of four balanced $2 \times 2$ MZIs, each including a thermal actuator to control the switching state. To simplify the analytical description, let us assume that all the actuators introduce equal thermal cross-talk on surrounding MZIs, so that the phase coupling matrix is

$$
\mathbf{T}=\left(\begin{array}{llll}
1 & \mu & \mu & \mu \\
\mu & 1 & \mu & \mu \\
\mu & \mu & 1 & \mu \\
\mu & \mu & \mu & 1
\end{array}\right) .
$$

Assuming a phase coupling ratio $\mu=0.18$, the eigensolutions of the phase coupling matrix $\mathbf{T}$ are: $\mathbf{P}_{1}=[-0.44-$ $\left.\begin{array}{lll}0.44 & 0.76 & 0.13\end{array}\right], \quad \mathbf{P}_{2}=\left[\begin{array}{llll}0.7 & -0.7 & 0 & 0\end{array}\right], \quad \mathbf{P}_{3}=$ $\left[\begin{array}{llll}0.22 & 0.22 & 0.40 & -0.85\end{array}\right], \quad \mathbf{P}_{4}=\left[\begin{array}{llll}0.5 & 0.5 & 0.5 & 0.5\end{array}\right] . \quad$ Phase change along the orthogonal transformed coordinates can be calculated as $\delta \boldsymbol{\Psi}=\mathbf{P}^{-1} \delta \boldsymbol{\Phi}$.

To show a numerical example, let us consider the reconfiguration of the $4 \times 4$ switch fabric to route an input optical signal ( $5 \mathrm{Gbit} / \mathrm{s}$ OOK), provided at port $I n 1$, to output port Out3. This path requires MZI1 to be set to "cross" state and MZI4 to be set to "bar" state. Random phase errors as large as $\pm \pi / 2$ are intentionally introduced in the unbalance of all the four MZIs of the PIC, leading to the 50 initial configurations shown in Fig. 4(b), which show the frequency domain transmission from $I n_{1}$ to $\mathrm{Out}_{3}$ (blue curves) and the optical crosstalk due to the path from $\mathrm{In}_{2}$ to $\mathrm{Out}_{3}$ (red curves). As in the example of Sec. III.A, we assume the same initial conditions and phase step-size $(0.05$ rad) for both TED-based tuning and individual tuning while maximizing the optical power at port Out3.

Numerical results show that, by using the TED-based algorithm, the switch fabric can be effectively steered to the desired configuration (see Fig. 4(c)), achieving less than $-25 \mathrm{~dB}$ optical crosstalk (red curves) for any initial conditions. It should be noted that convergence to almost identical transmission spectra is achieved even in cases where the initial switch state routes the signal almost entirely to other output ports. Histograms in Fig. 4(d) show that on average the TED-based algorithm requires less than 20 iterations to achieve convergence. In addition, the small standard deviation indicates that the number of required iterations is almost independent of the initial state of the switch. In contrast, if the MZIs are individually actuated (see Fig. 4(f)), the average number of iterations increases significantly and distributes randomly. This exhibits dependence on the initial state due to existence of

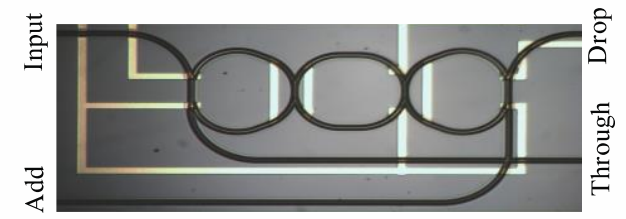

(a)

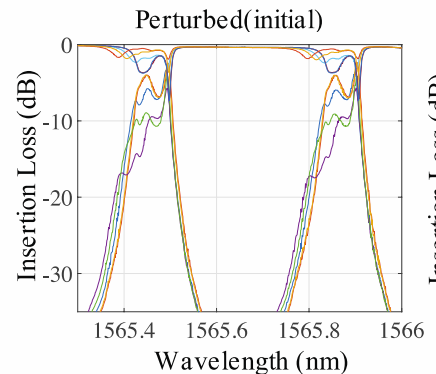

(b)

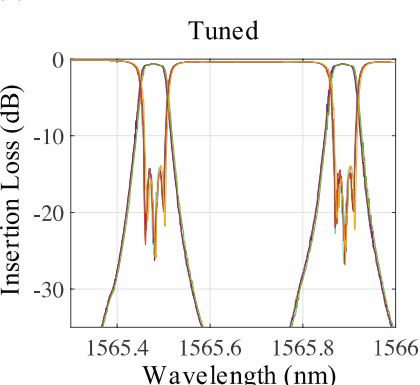

(c)

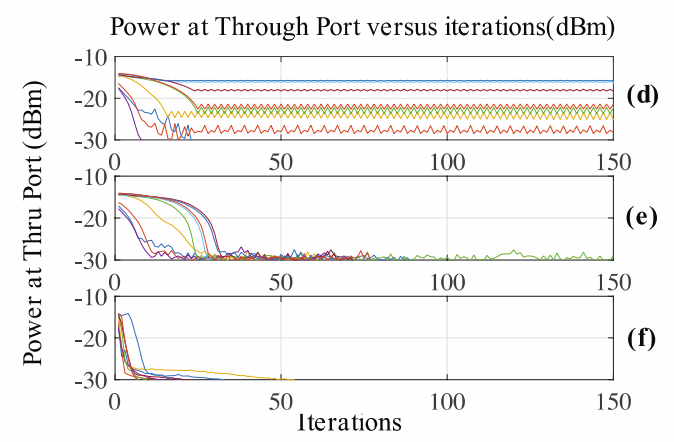

Fig. 5: Experimental validation of automatic tuning based on TED method. (a) Top view photograph of a $3^{\text {rd }}$ order coupled MRR filter fabricated in SiON technology. Measured transmission of the Through and Drop port of the filter (b) for five randomly perturbed configuration $(+/-100 \mathrm{pm})$ induced by using thermal phase shifters and (c) after automated tuning performed by using TED method. Convergence curves showing the measured optical power at Through port of the filter during the implementation of the following automatic tuning schemes: (d) individual tuning of MRRs, (e) TED-based tuning with fixed phase step and (f) TEDbased tuning with adaptive phase step.

thermal cross-talk. These results confirm the benefits of the TED method to ease the configuration of PICs in the presence of thermal crosstalk.

To assess the performance of the TED-based algorithm when the $\mathbf{T}$ matrix is not accurately known, the method was applied by intentionally underestimating the mutual phase perturbation (which is $\mu=0.18$ ) by $40 \%$, that is by considering $\mu=0.072$. While starting from the same initial points, non-efficient cancelation of thermal cross-talk is comprehensible by comparison between Figure 4(d) and (e). While comparing Figure 4(e) and (e) one can obviously assume that even TEDbased method with non-accurate phase coupling matrix is presenting more efficient convergence rates comparing to individual tuning. 


\section{EXPERIMENTAL RESULTS}

The effectiveness of the TED-based method has been experimentally validated on a $3^{\text {rd }}$ order coupled MRR filter. Fig. 5(a) shows a top view microphotograph of the device, which was fabricated in a high-index-contrast silicon oxynitride ( $\mathrm{SiON}$ ) photonic platform. The $4.4 \%$ refractive index contrast enables the realization of MRRs with a FSR of up to $100 \mathrm{GHz}$ with negligible bending loss. The SiON core channel waveguide has a square shape $\left(2.2 \times 2.2 \mu \mathrm{m}^{2}\right)$ and is buried in a silica cladding. More details on the waveguide design and fabrication process can be found in [26]. The round trip phase of each MRR of the filter can be individually controlled by means of metallic heater deposited on top of the waveguide uppercladding. The optical power is measured via an external photodiode and transmitted to a PC by ADC on USB interface. The control algorithm is implemented via software (Matlab) and the phase steps to be applied at each iteration (voltage change on each heater integrated in the PIC) are applied through a DAC on a USB interface.

In the following sections, the TED method is used to implement automatic tuning (Sec. IV.A) and wavelength locking (Sec. IV.B) of this device.

\section{A. Automatic tuning}

Phase perturbations were intentionally introduced in every MRR of the SiON filter by applying random errors in the voltages driving the heaters around their optimum tuning point. Figure 5(b) shows the measured Through and Drop port transmission of the perturbed filter when the induced frequency spread of the MRR resonances is as large as $100 \mathrm{pm}(12.5 \mathrm{GHz}$ versus $6.5 \mathrm{GHz} \mathrm{BW}$ of the filter). In line with the conditions considered in the numerical simulations of Sec. III.A, at the input port of the filter a $5 \mathrm{Gbit} / \mathrm{s}$ OOK modulated signal (with carrier wavelength of $1565.470 \mathrm{~nm}$ ) was used and the tuning algorithm was targeted to minimize the output power at the Through port. Assuming thermal crosstalk between the neighbor MRRs as the main contribution to the phase coupling (that is neglecting the phase coupling between the first and the third MRR), TED was implemented by using relations given in (6) and (7). In the experiments reported in this section, the temperature of the sample was kept constant at $25^{\circ} \mathrm{C}$ by using a thermoelectric-cooler (TEC) underneath the photonic sample. Figure 5(c) shows that, regardless of the initial perturbation, the filter was effectively tuned to the same shape, with a Through port isolation of $15 \mathrm{~dB}$ at convergence, corresponding to an estimated residual phase error of $\pi / 50$.

The TED method was then compared to the individual tuning in terms of convergence ratio and speed. For a fair comparison, we assumed the same perturbed configurations of Fig. 5(b) as the initial state of the filter and we applied both schemes with the same phase step-size for the heaters. As predicted by numerical simulations reported in Fig. 3(e), in many cases sequential tuning of individual resonators did not converge to the target filter shape and a poor Through port isolation with deep oscillations in the steady state was obtained. In contrast, the TED-based tuning did converge for all the considered initial cases in less than 40 iterations (see Fig. 5(e)). Since the TED method can be applied to generic algorithms, we
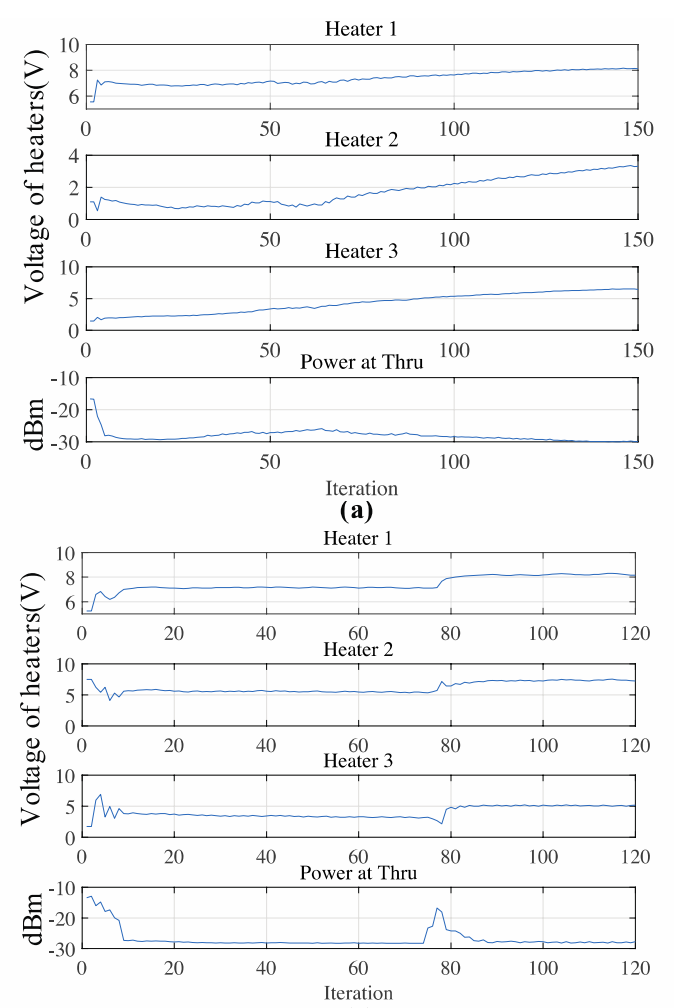

(b)

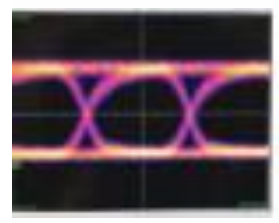

(c)

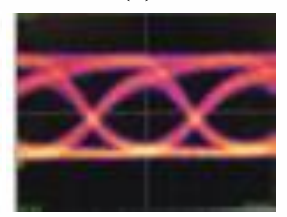

(d)

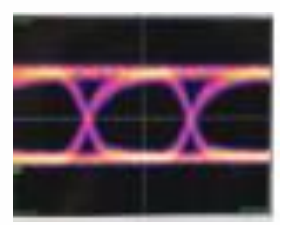

(e)
Fig. 6: Experimental validation of wavelength locking schemes based on TED method. Panels (a) and (b) show the voltages of heaters and the optical power measured at Through port of the filter when (a) automatic tuning and wavelength locking is performed while the temperature of the chip is changing by $3^{\circ} \mathrm{C}$ (35 pm shift) and (b) a sudden $30 \mathrm{pm}$ change in the wavelength of the input signal is introduced after iteration 75. (c) Reference eye diagram of the $5 \mathrm{Gbit} / \mathrm{s}$ OOK signal at the Drop port of the tuned filter. Panels (d) and (e) show the eye diagram after the introduction of $10 \mathrm{pm}$ shift of the channel wavelength when the wavelength locking algorithm is (d) off and (e) on.

also implemented an adaptive phase step-size technique to increase the convergence speed, while keeping the steady-state error unaffected. As an example, results in Fig. 5(f) show that convergence is accelerated by at least a factor 2 adapting the phase step-size of the heater according to the distance from the target point. A step size selection strategy that adaptively reduces the step size while progressing towards the target point allows for a faster convergence while reduces the perturbations and steady state error.

\section{B. Wavelength Locking}

We also investigated the possibility to exploit the TED-method with a wavelength locking algorithm to maintain the fine-tuned status of the filter (achieved though the automatic tuning procedure, see Fig. 5(c)) versus time varying perturbations of the system. These perturbations could be temperature fluctuations of the entire photonic chip as well as wavelength drifts of the input signal. 
In a first experiment, we demonstrate that TED-based tuning can efficiently work even though the temperature of the whole photonic sample is not stabilized. To this aim, we intentionally introduced a controlled temperature shift (linearly decreasing by $3^{\circ} \mathrm{C}$ ) by acting on the TEC underneath the sample, resulting in a wavelength shift of the transfer function of the filter by about $35 \mathrm{pm}$ (about $70 \%$ of the filter bandwidth). Figure 6(a) shows the evolution of heater voltages and Through port power during the automatic tuning of the filter, starting from a random initial condition of the MRR filter. From the comparison with the result of Fig. 5(e), where the temperature of the sample was kept constant by the TEC, we can conclude that: (i) temperature fluctuations do not significantly increase the number of iterations required to achieve convergence; (ii) once convergence to the target state of the filter is achieved (after about 10 iterations) the TED-method can be used to compensate against temperature fluctuations of the photonic sample (note that the voltages of the heaters increase, while the Through port power is almost unchanged).

Depending on the control algorithm adopted for the locking scheme, different values of wavelength drifts can be compensated. In the considered example of Fig. 6(b), we assumed a sudden drift in the wavelength of the input signal, as large as $30 \mathrm{pm}$ (equivalent to $3.75 \mathrm{GHz}$, that is about $60 \%$ of the filter bandwidth) which can be the result of using a look up table. This perturbation, which is responsible for the sharp increase (about $15 \mathrm{~dB}$ ) of the measured power at Through port, is completely recovered in less than 10 iterations.

Finally, we performed eye-diagram measurements to evaluate the performance of the TED-based schemes on the quality of transmitted signals. Figure 6(c) shows the reference eye diagram of the $5 \mathrm{Gbit} / \mathrm{s}$ OOK signal transmitted at the Drop port of the filter when the transfer function is tuned as in Fig. 5 (c) and the carrier wavelength of the signal $(1565.470 \mathrm{~nm})$ is aligned with the center of the filter passband. When the signal carrier wavelength is shifted by $10 \mathrm{pm}(20 \%$ of the filter bandwidth) and no wavelength locking scheme is employed, the eye diagram is distorted as shown in Fig. 5(d). In contrast, if the wavelength shift is applied when the wavelength locking algorithm is active (see Fig. 5(e)), no significant deterioration of the eye diagram is observed with respect to the reference signal.

\section{DISCUSSION}

In order to simplify the theoretical description of the TED method, in the examples presented in the previous sections, some assumptions were made on the phase coupling matrix $\mathbf{T}$ modelling the thermal crosstalk. However, the validity of the presented method can be extended to an arbitrary phase coupling matrix provided that (i) it is diagonalizable and (ii) its eigenvalues are all real. In fact, the eigenvalues of the $\mathbf{T}$ matrix are the elements of the diagonal matrix $\mathbf{T}_{\mathrm{D}}$ providing the relation between the desired and actual phase shift in the transformed phase space $\boldsymbol{\Psi}$. Both conditions are fulfilled if the T matrix is real and symmetric (with extension to spectral theorem [27]). In this case, an orthogonal matrix $\mathbf{P}$ exists, whose columns are the (real) eigenvectors of $\mathbf{T}$, such that $\boldsymbol{P}^{-1} \mathbf{T P}=\mathbf{T}_{D}$ is real diagonal.
This means that the TED method can be always applied if the phase coupling matrix is symmetric, that is if the thermal cross talk induced by the $m$-th heater on the $n$-th waveguide is equal to the crosstalk induced by the $n$-th heater on the $m$-th waveguide.

Since matrix symmetry is only a sufficient condition for diagonalization, the TED method could be applied also to a system described by a non-symmetric $\mathbf{T}$ matrix. However, designing the thermal actuators in order to guarantee equal thermal cross-talk among the integrated devices is a guideline that guarantees and simplifies the implementation of the TED method.

\section{CONCLUSION}

We theoretically introduced and experimentally demonstrated a technique capable of canceling the phase coupling due to thermal cross-talk in photonic integrated circuits. Instead of individually controlling actuators separately, in the TED technique all the actuators are controlled simultaneously according to appropriate weights, which are based on eigensolution of the thermally coupled system. Mathematically this implies a deterministic coordinate transformation, which can be applied to any kind of tuning and locking algorithm, based for instance on look-up tables [28], gradient-based [29] [30] and dithering-based techniques [23] [24], as well as multi degree of freedom (DOF) stabilization methods [21].

The use of the TED technique is not limited to specific circuit topologies but can be extended to generic PIC architectures. Through numerical simulations we proved its effectiveness in coupled MRR filters and in MZI switch fabrics. While in the individual control of phase actuators the thermal crosstalk can inhibits convergence, adopting the TED method convergence is always achieved in our simulations and experiments. Furthermore, the TED-based method allows to reduce the average number of required iterations while this number is also less sensitive to the initial perturbed state of the PIC. Even though complete thermal crosstalk cancelation would require precise knowledge of phase coupling matrix $\mathbf{T}$, we also demonstrated that the benefits of the TED method are mostly preserved when $\mathbf{T}$ is only partially known. This matrix can be approximated heuristically or obtained by thermal simulations, optical measurements or using data from implemented temperature sensors on the chip.

Experimental results performed on MRR-based filters confirm the faster and more robust convergence of the TEDbased tuning and locking algorithms, with respect to conventional approaches, to counteract temperature drifts or to track random fluctuations of the wavelength of the input signal.

Regarding the scalability of the proposed method, the complexity of any TED-based control algorithm scales linearly with the number of thermally coupled phase actuators. In contrast, if thermal crosstalk is not mitigated (e.g. techniques where heaters are individually controlled), complexity is expected to grow more than linearly, since additional steps are required to compensate for thermal cross-talk effects.

Finally, it should be noted that, although the TED method has been introduced here to cope with the specific problem of 
thermal crosstalk, its validity can be extended to other crosstalk effects, such as mechanical stress coupling in piezo actuators and RF coupling in transmission lines for instance in high-speed integrated optical modulators.

\section{ACKNOWLEDGMENT}

This work was (partially) performed at Polifab, the microand nanofabrication facility of Politecnico di Milano (www.polifab.polimi.it).

\section{REFERENCES}

[1] Y. Shen, N. C. Harris, S. Skirlo, M. Prabhu, T. Baehr-Jones, M. Hochberg, X. Sun, S. Zhao, . H. Larochelle, . D. Englund and M. Soljačić, "Deep learning with coherent nanophotonic circuits," Nature Photonics, vol. 11, p. 441 EP, 2017.

[2] D. Pérez, I. Gasulla, L. Crudgington, D. J. Thomson, A. Z. Khokhar, K Li, W. Cao, G. Z. Mashanovich and J. Capmany, "Multipurpose silicon photonics signal processor core," Nature Communications, vol. 8, p. 636, 2017.

[3] J. Carolan, C. Harrold, C. Sparrow, E. Martín-López, N. J. Russell, J. W. Silverstone, P. J. Shadbolt, N. Matsuda, M. Oguma, M. Itoh, G. D. Marshall, M. G. Thompson, J. C. F. Matthews, T. Hashimoto, J. L. O’Brien and A. Laing, "Universal linear optics," OpticsScience, vol. 349, no. 6249, pp. 711-716, 2015.

[4] L. Zhuang, C. G. H. Roeloffzen, M. Hoekman, K.-J. Boller and A. J. Lowery, "Programmable photonic signal processor chip for radiofrequency applications," Optica, vol. 2, no. 10, pp. 854-859, 2015.

[5] F. Xia, L. Sekaric and Y. Vlasov, "Ultracompact optical buffers on a silicon chip," Nature Photnics, pp. 65-71, 2007.

[6] Z. Lu, J. Jhoja, J. Klein, X. Wang, A. Liu, J. Flueckiger, J. Pond and L. Chrostowski, "Performance prediction for silicon photonics integrated circuits with layout-dependent correlated manufacturing variability," Optics Express, vol. 25, no. 9, pp. 9712-9733, 2017.

[7] S. Grillanda, S. Fu, R. Ji, F. Morichetti, N. Peserico, I. Belladelli, M. Carminati, G. Ferrari, M. Sampietro, A. Dentin, A. Dedè, A. Vannucci, B. Holmes, C. Klitis and So, "Wavelength Locking Platform for DMLbased Multichannel Transmitter on a Silicon Chip," in Optical Fiber Communication Conference, 2016.

[8] C. Sun, M. Wade, M. Georgas, S. Lin, L. Alloatti, B. Moss, R. Kumar, A. H. Atabaki, F. Pavanello, J. M. Shainline, J. S. Orcutt, R. J. Ram, M. Popović and V. Stojanović, "A $45 \mathrm{~nm}$ CMOS-SOI Monolithic Photonics Platform With Bit-Statistics-Based Resonant Microring Thermal Tuning," Journal of Solid-State Circuits, vol. 51, no. 4, 2016.

[9] X. Zheng, E. Chang, P. Amberg, I. Shubin, J. Lexau, F. Liu, H. Thacker, S. S. Djordjevic, S. Lin, Y. Luo, J. Yao, . J.-H. Lee, K. Raj, R. Ho, J. E. Cunningham and A. V. Krishnamoorthy, "A high-speed, tunable silicon photonic ring modulator integrated with ultra-efficient active wavelength control," Optics Express, vol. 22, no. 10, pp. 1262812633, 2014.

[10] H. Jayatilleka, K. Murray, M. Á. Guillén-Torres, M. Caverley, R. Hu, N. A. F. Jaeger, L. Chrostowski and S. Shekhar, "Wavelength tuning and stabilization of microring-based filters using silicon in-resonator photocond," Opt. Express, vol. 23, no. 19, pp. 25084-25097, 2015.

[11] H. Jayatilleka, H. Shoman, R. Boeck, N. A. F. Jaeger, L. Chrostowski and S. Shekhar, "Automatic Configuration and Wavelength Locking of Coupled Silicon Ring Resonators," Journal of Lightwave Technology, vol. 36, pp. 210-218, 2018.

[12] J. C. C. Mak, W. D. Sacher, T. Xue, J. C. Mikkelsen, Z. Yong and J. Poon, "Automatic Resonance Alignment of High-Order Microring Filters," Quantum Electronics, vol. 51, no. 11, pp. 1-11, 2015.

[13] Y. Li and A. W. Poon, "Actively stabilized silicon microrings with integrated surface-state-absorption photodetectors using a slopedetection method," Optical express, vol. 24, no. 19, pp. 21286-21300, 2016.
[14] N. C. Harris, Y. Ma, J. Mower, T. Baehr-Jones, D. Englund, M. Hochberg and C. Galland, "Efficient, compact and low loss thermooptic phase shifter in silicon," Optics Express, vol. 22, no. 9, pp. 1048710493, 2014.

[15] P. DasMahapatra, R. Stabile, A. Rohit and K. A. Williams, "Optical Crosspoint Matrix Using Broadband Resonant Switches," IEEE Journal of Selected Topics in Quantum Electronics, vol. 20, no. 4, pp. 1--10, 2014.

[16] Po Dong, Wei Qian, Hong Liang, Roshanak Shafiiha, Ning-Ning Feng, Dazeng Feng, Xuezhe Zheng, Ashok V. Krishnamoorthy, and Mehdi Asghari, "Low power and compact reconfigurable multiplexing devices based on silicon microring resonators," Opt. Express, vol. 18, no. 10, pp. 9852-9858, 2010.

[17] B. Guha, J. Cardenas and M. Lipson, "Athermal silicon microring resonators with titanium oxide cladding," Opt. Express, vol. 21, no. 22, pp. 26557-26563, 2013.

[18] S. Djordjevic, K. Shang, B. Guan, S. Cheung, L. Liao, J. Basak, H.-F. Liu and S. J. B. Yoo, "CMOS-compatible, athermal silicon ring modulators clad with titanium dioxide," Opt. Express, vol. 21, no. 12, pp. 13958-13968, 2013.

[19] L. Lu, L. Zhou, X. Sun, J. Xie, Z. Zou, H. Zhu, X. Li and J. Chen, "CMOS-compatible temperature-independent tunable silicon optical lattice filters," Opt. Express, vol. 21, no. 8, pp. 9447-9456, 2013.

[20] S. Dwivedi, H. D'heer and W. Bogaerts, "A Compact All-Silicon Temperature Insensitive Filter for WDM and Bio-Sensing Applications," Photonics Technology Letters, vol. 25, no. 22, pp. 21672170, 2013.

[21] F. Jonathan , A. Kodanev and M. Nazarathy, "Multi-Degree-ofFreedom Stabilization of Large-Scale Photonic-Integrated Circuits," Journal of Lightwave Technology, vol. 33, no. 10, pp. 2146-2166, 2015.

[22] R. L. Williams and D. A. Lawrence, Linear State-Space Control Systems, Hoboken NJ, USA: Wiley, 2007.

[23] K. Padmaraju, D. F. Logan, T. Shiraishi, J. J. Ackert, A. P. Knights and K. Bergman, "Wavelength Locking and Thermally Stabilizing Microring Resonators Using Dithering Signals," Journal of Lightwave Technology, vol. 32, no. 3, pp. 505-512, 2014.

[24] K. Padmaraju, L.-W. Luo, X. Zhu, M. Glick, R. Dutt, M. Lipson and K. Bergman, "Wavelength locking of a WDM silicon microring demultiplexer using dithering signals," in Optical Fiber Conference, an Francisco, CA, USA, 2014.

[25] S. Grillanda, F. Morichetti, N. Peserico, P. Ciccarella, A. Annoni, M. Carminati and A. Melloni, "Non-Invasive Monitoring of ModeDivision Multiplexed Channels on a Silicon Photonic Chip," Journal of Lightwave Technology, vol. 33, no. 6, pp. 1197-1201, 2015.

[26] A. Melloni, F. Morichetti, G. Cusmai, R. Costa, A. Breda, C. Canavesi, and M. Martinelli, "Progress in large integration scale circuits in SiON technology," in Transparent Optical Networks, Rome. Italy, 2007.

[27] Watkins, David S., Fundamentals of Matrix Computations, John Wiley \& Sons, 2004.

[28] A. Mahendra, C. Xiong, X. Zhang, B. Eggleton and P. Leong, "Multiwavelength stabilization control of a thermo-optic system with adaptive reconfiguration," Applied Optics, vol. 56, no. 4, p. 1113, 2017.

[29] P. Dong, R. Gatdula, K. Kim, J. H. Sinsky, A. Melikyan, Y.-K. Chen, G. de Valicourt and J. Lee, "Simultaneous wavelength locking of microring modulator array with a single monitoring signa," Optics Express, vol. 25, no. 14, pp. 16040-16046, 2017.

[30] A. Gazman, C. Browning, Z. Zhu, L. P. Barry and K. Bergman, "Automated Thermal Stabilization of Cascaded Silicon Photonic Ring Resonators for Reconfigurable WDM Applications," in European Conference on Optical Communication (ECOC), Gothenburg, Sweden, 2017. 